\title{
Domain-specific Modelling with AToM $^{3}$
}

\author{
Hans Vangheluwe, Ximeng Sun, Eric Bodden \\ School of Computer Science, McGill University \\ Montréal, Québec, Canada \\ $\{$ hv, xsun16, ebodde\}@cs.mcgill.ca
}

\begin{abstract}
Using domain-specific modelling environments maximally constrains users, matching their mental model of the problem domain, and allows them to only build syntactically correct models. Anecdotal evidence shows that domainspecific modelling can drastically improve productivity as well as product quality. In this paper, the foundations of (domain-specific) modelling language design are presented. Our guiding principle is to "model everything". It is indeed shown how all aspects of language design can be explicitly (meta-)modelled enabling the efficient synthesis of domain-specific, visual, modelling environments. The case of $\mathrm{AToM}^{3}$, A Tool for Multi-formalism and Meta Modelling, is elaborated. Concepts are illustrated by modelling, analysis, simulation, and eventual synthesis of software for Traffic networks.
\end{abstract}

\section{Dissecting a Modelling Language}

To explicitly model domain-specific modelling languages and ultimately synthesize visual modelling environments for those, we will break down a modelling language into its basic constituents. The following is based on a description by Harel and Rumpe [1], taking common programming language concepts and putting them in a more general modelling context. An earlier version of this section appeared as a tutorial at a 2006 MoDELS workshop [2].

The two main aspects of a model are its syntax (how it is represented) on the one hand and its semantics (what it means) on the other hand.

The syntax of modelling languages is traditionally partitioned into concrete syntax and abstract syntax. In textual languages for example, the concrete syntax is made up of sequences of characters taken from an alphabet. These characters are typically grouped into words or tokens. Certain sequences of words or sentences are considered valid (i.e., belong to the language). The (possibly infinite) set of all valid sentences is said to make up the language. Costagliola et. al. [3] present a framework of visual language classes in which the analogy between textual and visual characters, words, and sentences becomes apparent. Visual languages are those languages whose concrete syntax is visual (graphical, geometrical, topological, ...) as opposed to textual. For practical reasons, models are often stripped of irrelevant concrete syntax information during syntax checking. This results in an "abstract" representation which captures the "essence" of the model. This is called the abstract syntax. Obviously, a single abstract syntax may be represented using multiple concrete syntaxes. In programming language compilers, abstract syntax of models (due to the nature of programs) is typically represented in 
Abstract Syntax Trees (ASTs). As in the context of general modelling, models are often graph-like, this representation can be generalized to Abstract Syntax Graphs (ASGs). Once the syntactic correctness of a model has been established, its meaning must be specified. This meaning must be unique and precise (to allow correct model exchange and code synthesis for example). Meaning can be expressed by specifying a semantic mapping function which maps every model in a language onto an element in a semantic domain. For example, the meaning of Activity Diagrams may be given by mapping it onto Petri Nets. For practical reasons, semantic mapping is usually applied to the abstract rather than to the concrete syntax of a model. Note that the semantic domain is a modelling language in its own right which needs to be properly modelled (and so on, recursively). In practice (in tools), the semantic mapping function maps abstract syntax onto abstract syntax.

To continue this introduction of meta-modelling and model transformation concepts, languages will explictly be represented as (possibly infinite) as shown in Figure 1. In the figure, insideness denotes the sub-set relationship. The dots represent models

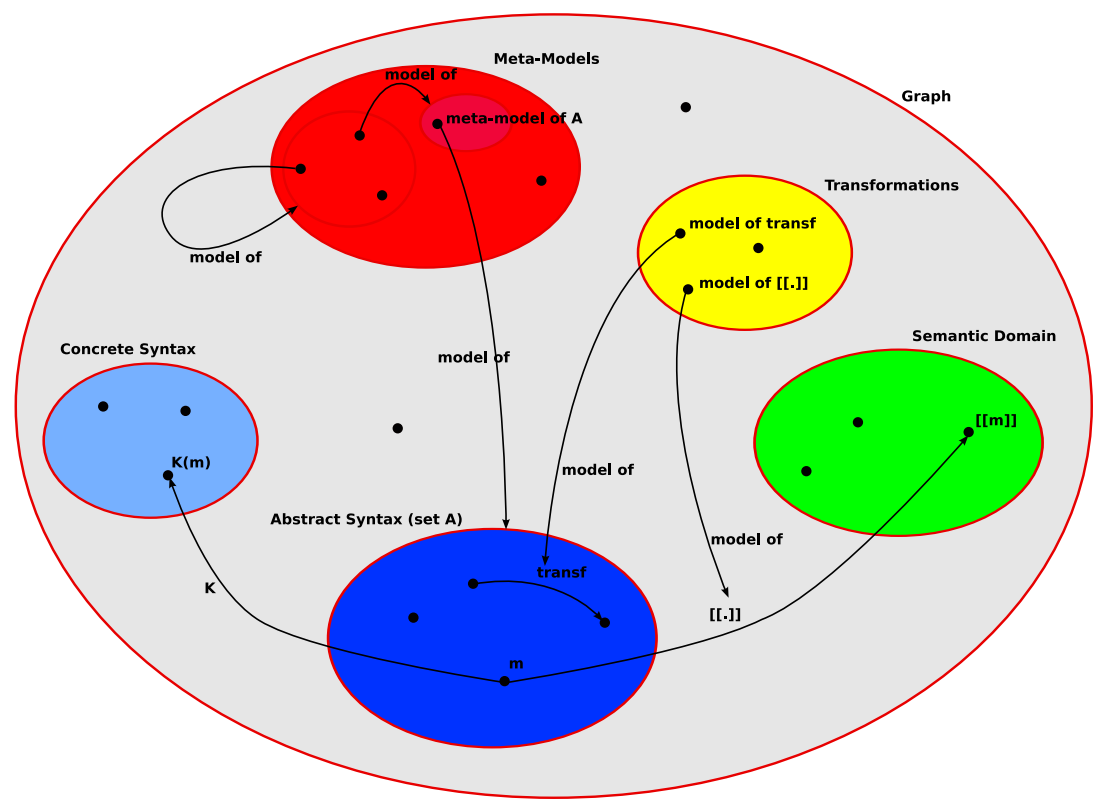

Fig. 1. Modelling Languages as Sets

which are elements of the encompassing set(s). As one can always, at some level of abstraction, represent a model as a graph structure, all models are shown as elements of the set of all graphs Graph. Though this restriction is not necessary, it is commonly used as it allows for the elegant design, implementation and bootstrapping of (meta-)modelling environments. As such, any modelling language becomes a (possibly infinite) set of graphs. In the bottom centre of Figure 1 is the abstract syntax set A. It is a set of models stripped of their concrete syntax. 
Meta-modelling is a heavily over-used term. Here, we will use it to denote the explicit description (in the form of a finite model in an appropriate meta-modelling language) of the abstract syntax set $A$ of a modelling language. Often, meta-modelling also covers a model of the concrete syntax. Semantics is however not covered. In the figure, the set $A$ is described by means of the model meta-model of $A$. On the one hand, a meta-model can be used to check whether a general model (a graph) belongs to the set $A$. On the other hand, one could, at least in principle, use a meta-model to generate all elements of $A$. This explains why the term meta-model and grammar are often used inter-changeably.

Several languages are suitable to describe meta-models. Two approaches are in common use:

1. A meta-model is a type-graph. Elements of the language described by the metamodel are instance graphs. There must be a morphism between an instance-graph (model) and a type-graph (meta-model) for the model to be in the language. Commonly used meta-modelling languages are Entity Relationship Diagrams (ERDs) and Class Diagrams (adding inheritance to ERDs). The expressive power of this approach is often not sufficient and an extra constraint language (such as the $\mathrm{Ob}$ ject Constraint Language (OCL) in the UML) specifying constraints over instances is used to further specify the set of models in a language (adding the expressive power of first or higher order logic). This is the approach used by the OMG to specify the abstract syntax of the UML.

2. An alternative general approach specifies a meta-model as a transformation (in an appropriate formalism such as Graph Grammars [4]) which, when applied to a model, verifies its membership of a formalism by reduction. This is similar to the syntax checking based on (context-free) grammars used in programming language compiler compilers. Note how this approach can be used to model type inferencing and other more sophisticated checks.

Both types of meta-models (type-graph or grammar) can be interpreted (for flexibility and dynamic modification) or compiled (for performance). Note that when metamodelling is used to synthesize interactive, possibly visual modelling environments, we need to model when to check whether a model belongs to a language. In free-hand modelling, checking is only done when explicitly requested. This means that it is possible to create, during modelling, syntactically incorrect models. In syntax-directed modelling, syntactic constraints are enforced at all times during editing to prevent a user from creating syntactically incorrect models. Note how the latter approach, though possibly more efficient, due to its incremental nature -of construction and consequently of checkingmay render certain valid models in the modelling language unreachable through incremental construction. Typically, syntax-directed modelling environments will be able to give suggestions to modellers whenever choices with a finite number of options present themselves.

The advantages of meta-modelling are numerous. First, an explicit model of a modelling language can serve as documentation and as specification. Such a specification can be the basis for the analysis of properties of models in the language. From the metamodel, a modelling environment may be automatically generated. The flexibility of the approach is tremendous: new, possibly domain-specific, languages can be designed by 
simply modifying parts of a meta-model. As this modification is explicitly applied to models, the relationship between different variants of a modelling language is apparent. Above all, with an appropriate meta-modelling tool, modifying a meta-model and subsequently generating a possibly visual modelling tool is orders of magnitude faster than developing such a tool by hand. The tool synthesis is repeatable and less error-prone than hand-crafting. As a meta-model is a model in an appropriate modelling language in its own right, one should be able to meta-model that language's abstract syntax too. Such a model of a meta-modelling language is called a meta-meta-model. This is depicted in Figure 1. It is noted that the notion of "meta-" is relative. In principle, one could continue the meta- hierarchy ad infinitum. Luckily, some modelling languages can be meta-modelled by means of a model in the language itself. This meta-circularity allows modelling tool and language compiler builders to bootstrap their systems.

A model $\mathrm{m}$ in the Abstract Syntax set (see Figure 1) needs at least one concrete syntax. This implies that a concrete syntax mapping function $\kappa$ is needed. $\kappa$ maps an abstract syntax graph onto a concrete syntax model. Such a model could be textual (e.g., an element of the set of all Strings), or visual (e.g., an element of the set of all the $2 \mathrm{D}$ vector drawings). Note that the set of concrete models can be modelled in its own right. It is noted that grammars may be used to model a visual concrete syntax [5]. Also, concrete syntax sets will typically be re-used for different languages. Often, multiple concrete syntaxes will be defined for a single abstract syntax, depending on the intended user. If exchange between modelling tools is intended, an XML-based textual syntax is appropriate. If in such an exchange, space and performance is an issue, a binary format may be used instead. When the formalism is graph-like as in the case of a circuit diagram, a visual concrete syntax is often used for human consumption. The concrete syntax of complex languages is however rarely entirely visual. When for example equations need to be represented, a textual concrete syntax is more appropriate.

Finally, a model $m$ in the Abstract Syntax set (see Figure 1) needs a unique and precise meaning. This is achieved by providing a Semantic Domain and a semantic mapping function [ [.]]. This mapping can be given informally in English, pragmatically with code or formally with model transformations. Natural languages are ambiguous and not very useful since they cannot be executed. Code is executable, but it is often hard to understand, analyze and maintain. It can be very hard to understand, manage and derive properties from code. This is why formalisms such as Graph Grammars are often used to specify semantic mapping functions in particular and model transformations in general. Graph Grammars are a visual formalism for specifying transformations. Graph Grammars are formally defined and at a higher level than code. Complex behavior can be expressed very intuitively with a few graphical rules. Furthermore, Graph Grammar models can be analyzed and executed. As efficient execution may be an issue, Graph Grammars can often be seen as an executable specification for manual coding. As such, they can be used to automatically generate transformation unit tests.

Not only semantic mapping, but also general model transformations can be explicitly modelled as illustrated by -transf - and its model in Figure 1. It is noted that models can be transformed between different formalisms.

Within the context of this paper, we have chosen to use the following terminology (see also [2]). 
- A language is the set of abstract syntax models. No meaning is given to these models.

- A concrete language comprises both the abstract syntax and a concrete syntax mapping function $\kappa$. Obviously, a single language may have several concrete languages associated with it.

- A formalism consists of a language, a semantic domain and a semantic mapping function giving meaning to model in the language.

- A concrete formalism comprises a formalism together with a concrete syntax mapping function.

We will also focus on our tool $\mathrm{AToM}^{3}$ [6]. It is noted that several other meta-environment toolsets exists (see for example www.meta-environment.org). We use our tool as it closely follows the general framework described above.

Many challenges still remain for Model Driven Engineering. As with programs, models evolve over time. Model version control, based on computing model differences is necessary. As even meta-models and models transformations (in particular, of semantics) may evolve, this must also be dealt with.

\section{Modelling Traffic Networks}

Domain- and formalism-specific modelling have the potential to greatly improve productivity [7]. They are able to exploit features inherent to a specific domain or formalism. This will for example enable specific analysis techniques or the synthesis of efficient code. The time required to construct such domain/formalism-specific modelling and simulation environments can however be prohibitive. Thus, rather than using such specific environments, generic environments are typically used. Those are necessarily a compromise.

To illustrate domain-specific modelling, we introduce a simplified TimedTraffic formalism, a visual notation specific to the vehicle traffic domain [8]. It is of course possible to model traffic systems using a variety of generic modelling and simulation languages such as GPSS, DEVS [9], and Petri Nets. We choose not to do this, but rather build a TimedTraffic-specific modelling environment. This maximally constrains users, allowing them, by construction, to only build syntactically and, for as far as this can be statically checked, semantically correct models. Furthermore, the TimedTraffic-specific, visual syntax used matches the users' mental model of the problem domain.

Figure 2 shows a lattice of traffic-related Formalisms and relevant transformations between them. At the top is TimedTraffic, a domain-specific formalism allowing the description of timed movement of cars through a traffic network. A modeller may wish to visualize the dynamics of a traffic systems, analyze properties such as liveness, and obtain performance metrics such as average throughput. To support this variety of goals, Figure 2 shows how TimedTraffic is mapped onto different formalisms. When timing information is removed from a model, a conservative abstraction, an untimed Traffic model is obtained. As shown in [10], an appropriate transformation onto Petri Nets then allows for analysis of pertinent properties such as liveness and conservation. For timed analysis, mapping onto Timed Transition Petri Nets may be done. For performance analysis by means of simulation, mapping onto the DEVS formalism (and simulation using for example the pythonDEVS tool) is appropriate. Although desirable, imple- 


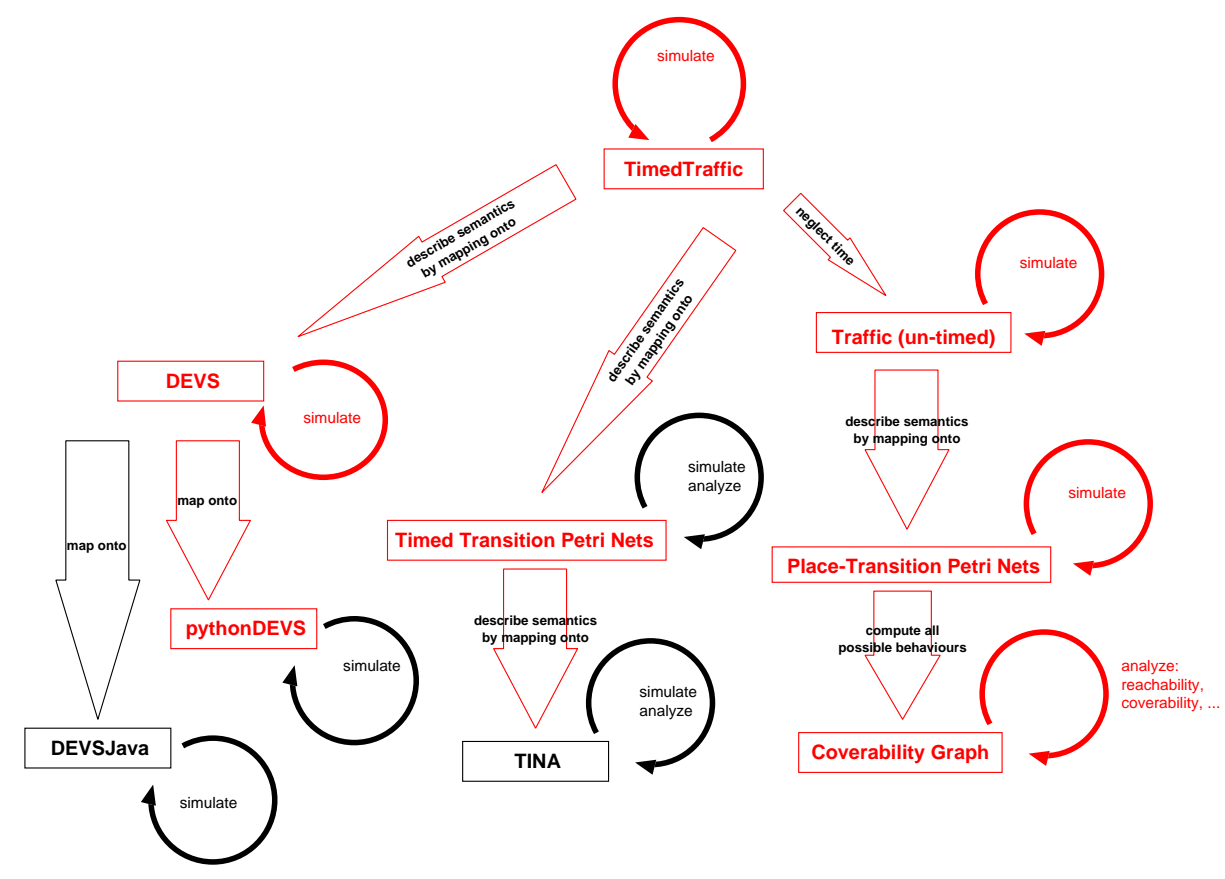

Fig. 2. Various Traffic formalisms and transformations between them

menting modelling environments which support the formalisms and transformations in Figure 2 seems a daunting task. The meta-modelling and model transformation concepts described in the previous section can however be used to model all formalisms and transformations. We have implemented the entire figure but for brevity will demonstrate the principles of our approach by showing the meta-model and the operational semantics of TimedTraffic.

\subsection{Modelling TimedTraffic}

A modelling environment for the domain-specific formalism TimedTraffic allows users to model traffic flow by means of connected road segments, cars and traffic lights. Traffic signals impose constraints on how cars can be moved by this transformation. Furthermore, the model is timed, i.e., cars move at a certain constant speed (in our chosen abstraction) and traffic lights switch state every fixed number of time units.

We first introduce the abstract syntax of TimedTraffic and explain how it can be modelled within the $\mathrm{AToM}^{3}$ modelling environment, along with the static semantics which imposes certain non-behavioural constraints. We then show how concrete syntax information can be added to allow synthesis of a visual modelling tool for TimedTraffic. We also demonstrate how concrete syntax can change over time to reflect state changes on the abstract level. We finally model (operational) semantics of this formalism by means of a graph grammar which describes how cars move through a given traffic network.

Abstract Syntax The abstract syntax model or meta-model of TimedTraffic is shown as a model in the Entity Relationship formalism in Figure 3. This ER meta-model com- 


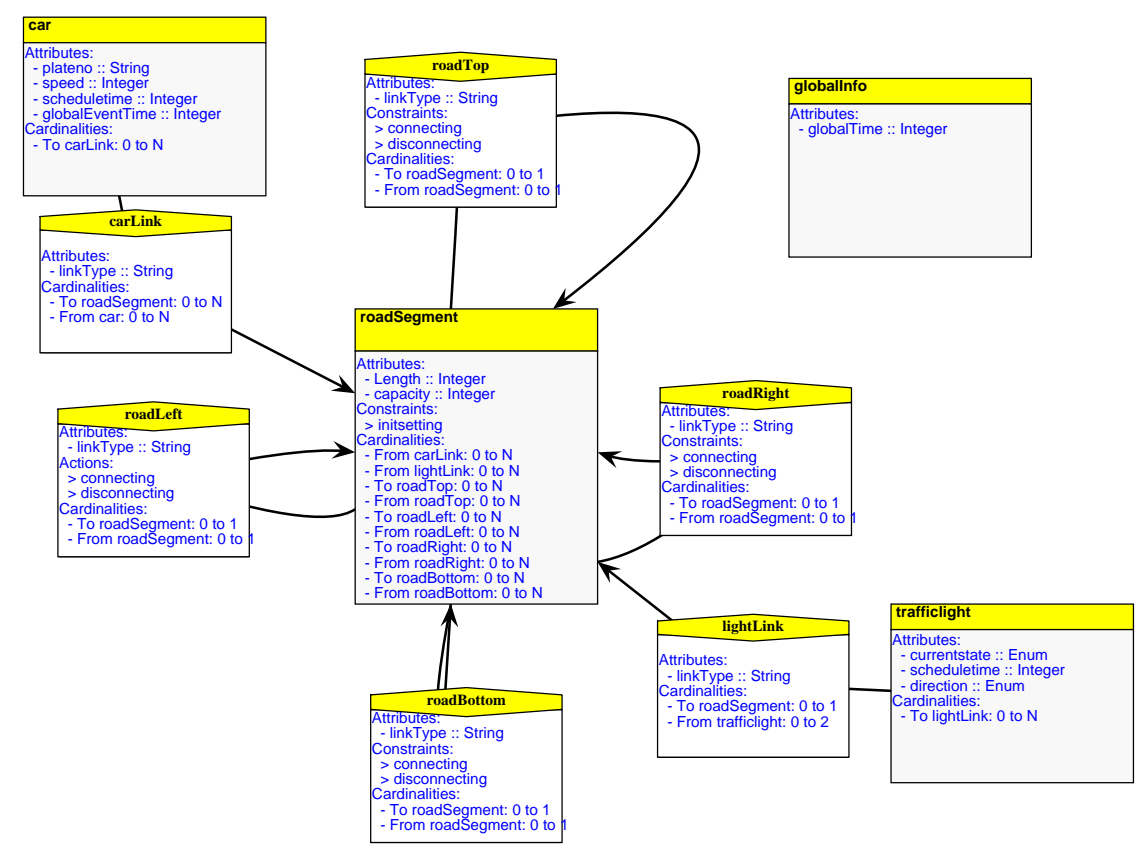

Fig. 3. Metamodel for the abstract syntax of Timed traffic

prises the following entities and relations:

roadSegment Can be connected to other road segments. Also, since our model is a timed model, road segments have a size to determine the time a car needs to cross them. A road segment may also have a finite capacity.

car Each car can move almost independently through the network. "Almost" because a car is not allowed to cross a road segment which has a connected traffic light showing red. A car has a certain fixed speed and based on this and the length of a road segment, a "schedule time" can be computed which gives the time of its next move, relative to the last move time. For informational purposes, we also include a global event time, which shows the global timestamp of the next scheduled move.

trafficLight A traffic light in our simple model can have two states, red and green. Just like cars, they have a schedule time, which reflects the time of their next state change.

globalInfo This is singleton global entity for informational purposes showing the global time during the simulation.

relations There are multiple types of associations which indicate which entities may be connected. These impose multiplicity constraints: a road segment can only be connected to at most one other road segment per direction (top, bottom, left, right). This is enforced by setting cardinalities To roadSegment: 0 to 1 and From roadSegment: 0 to 1.

The abstract syntax induced by this metamodel rigorously defines all entities, their attributes, their possible connections and constraints amongst them. The user of the modelling environment for TimedTraffic is however probably more concerned with the concrete syntax. In the following, we show how such a concrete syntax can be given to each abstract counterpart using $\mathrm{AToM}^{3}$. 
Concrete Syntax Cars are rendered as a simple icon (constructed in $\mathrm{AToM}^{3}$ 's icon editor drawing tool) showing a bird's eye view of a car. On top the car's global move time is displayed. See Fig. 6 for an example. Road segments, as mentioned above, can be connected to zero to one other road segments on each side. To reflect which each road segment is connected to, each such segment contains four arrows, each of which is made visible when the segment is being connected and made invisible on disconnection. The effect of this is seen in Fig. 6, where only those arrows are visible that relate to existing connections. In addition to the concrete syntax (an icon) for each entity, a concrete syntax needs to be associated with each association. Two types of concrete syntax are typically used. On the one hand, associations can be rendered by means of geometric constraints. Connected road segments will for example be visually placed next to one another. On the other hand, a spline with a pointed arrow may be used as in the case of the connection between a traffic light and a road segment. A traffic light is modelled by a traffic light icon along with two textual labels showing the current state and the number of time units until the next state switch. The actual switch is triggered by a graph grammar action as described below.

One concrete instance of a TimedTraffic model is shown in figure 6 which also shows how it evolves over time. This behaviour is modelled in a graph grammar which we explain in the following section.

Operational Semantics (Behaviour) The transformation of models is a crucial element in all model-based endeavours. As models, meta-models, and meta-meta-models are all in essence attributed, typed graphs, we can transform them by means of graph rewriting. The rewriting is specified in the form of Graph Grammar [?] models. These are a generalization, for graphs, of Chomsky grammars. They are composed of rules. Each rule consists of Left Hand Side (LHS) and Right Hand Side (RHS) graphs. Rules are evaluated against an input graph, called the host graph. If a matching is found between the LHS of a rule and a sub-graph of the host graph, then the rule can be applied. When a rule is applied, the matching subgraph of the host graph is replaced by the RHS of the rule. Rules can have applicability conditions, as well as actions to be performed when the rule is applied. Some graph rewriting systems have control mechanisms to determine the order in which rules are checked. After a rule matching and subsequent application, the graph rewriting system starts the search again. The graph grammar execution ends when no more matching rules are found.

The behaviour of any syntactically valid TimedTraffic model is given by a set of Graph Grammar rules. Each car has an initial "next move time". After each move (caused by a graph grammar rule) we recalculate the next move time based on the car's speed attribute and the length attribute of the road segment that has been moved to. We can thus calculate the next move time of each car:

$$
\text { car.nextMoveTime }=\frac{\text { targetRoadSegment.length }}{\text { car.speed }}
$$

Note that this move time is relative: it gives the number of time units until its next move. Consequently, a car can be moved whenever its next move time is zero (unless it is blocked due to a red light). The rule in Fig. 4 reflects this transformation when a car is moved to the right. The rule has a condition (not shown here) that the schedule time 
1. turn light (red/green)

2. move car (left/right/up/down)

3. reschedule

This leads to the fact that first all traffic lights with a schedule time of 0 are switched. If there is no such light or all have been switched already, then all cars with a schedule time of 0 are moved. Since all "move" rules have the same priority, the move direction is random (within the constraints given by connected red traffic lights). Finally, when all such cars are moved we know that no light or car with schedule time 0 exists any more and hence we can safely advance time by applying (i.e., matching) the last rule.

Example Simulation An example of applying some steps of the transformation is shown in Figure 6 (to be read top to bottom, left to right). We start with the traffic light showing green and a schedule time of 10 time units. The left car (with a speed of 10) is about to move and the right car (with a speed of 3 ) is to be moved in 4 time units. The size of each road segment is 40. According to
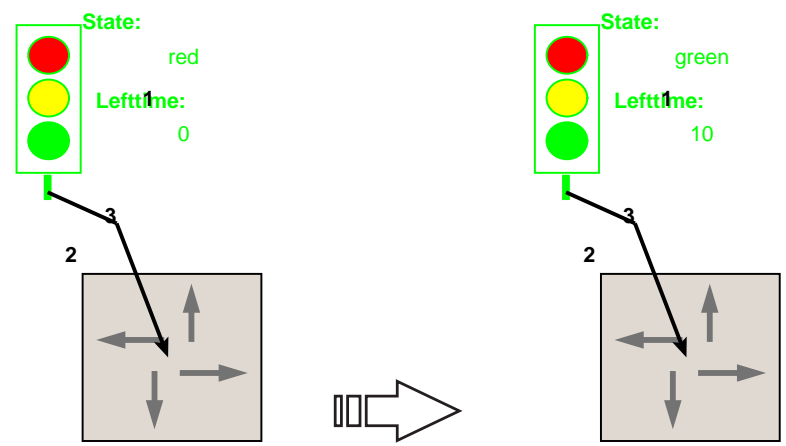

Fig. 5. Light behaviour

the priorities stated above, the first match we obtain is the rule moving the left car to the right, since its schedule time is 0 . Afterwards, only the last rule can be applied and hence we reschedule, which leads to the third subfigure. Note that the new schedule time for the moved car is again 4 by equation (1). In this state, both cars can move since for both the move time is 4 , which equals the global time (i.e., for both, the offset is 0 and one of the "move" rules can apply). Here, nondeterministically, the left car is chosen and is moved back again, leading to the fourth sub-figure. The next move time for this car is now 8 , by equation (1). In the next step, the second car is moved to the left and also its next move time is recalculated. According to the same equation, this time we calculate a time of 17 , adding $40 / 3=13$ to the previous move time 4 . Then, no more cars can move and so time is incremented by applying the "reschedule" rule. After doing so, the schedule time of the traffic light becomes 0 which means that in the subsequent step, the state of this light can be switched to "red". Then again time passes until the global time 17 (one but last subfigure) which enables the second car to move. This simulation could be executed over an arbitrary amount of time and indeed AToM ${ }^{3}$ allows for a continuous application of transformations.

\section{Conclusions}

In this paper, we demonstrated the use of meta-modelling and graph transformation for domain-specific modelling. We visually specify the abstract syntax (meta- 

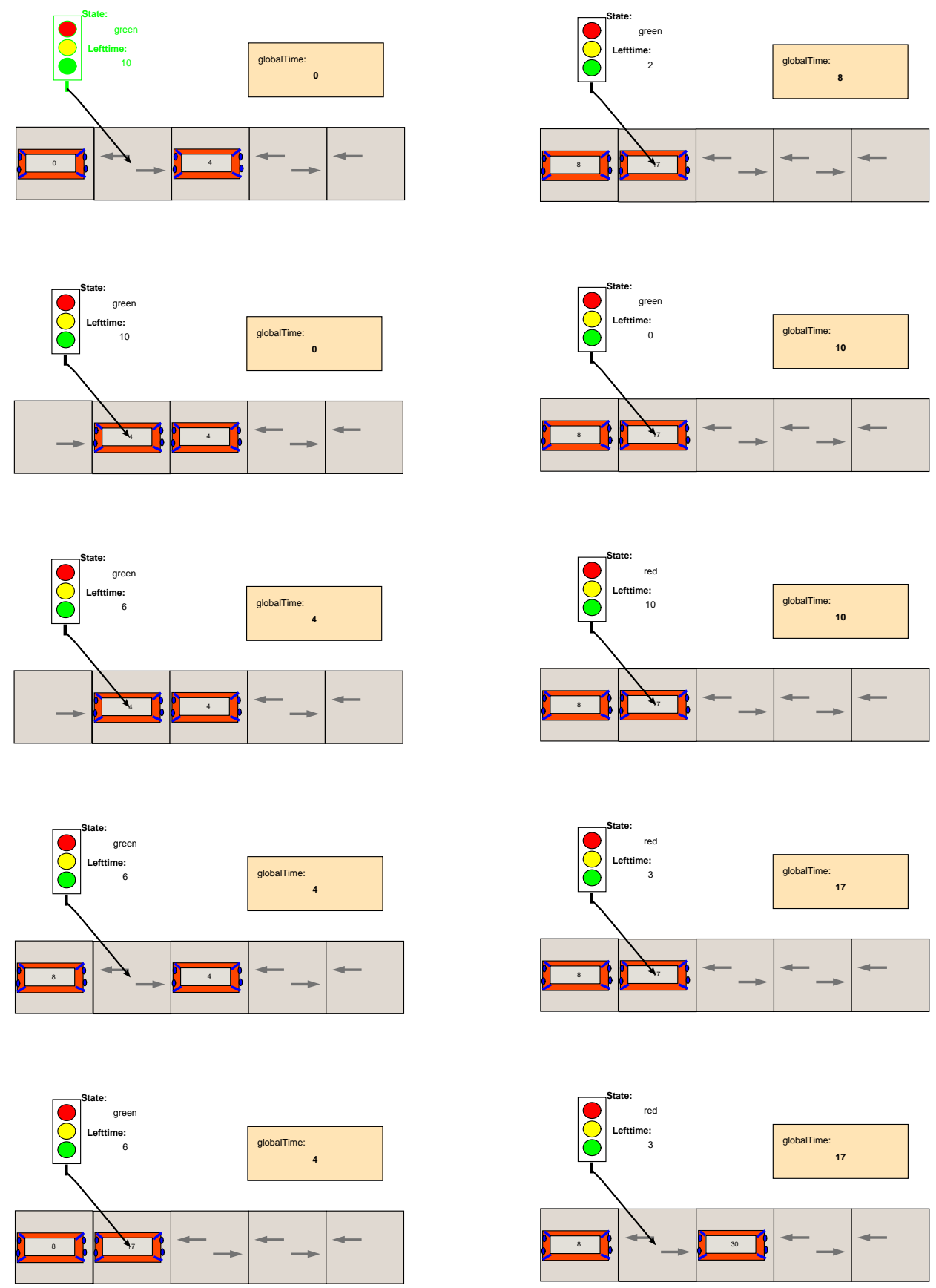

Fig. 6. Resulting simulation trace/animation 
modelling) and concrete syntax of models we want to deal with. By means of graph transformation we visually define the manipulations on these models. This has the advantage that transformations are explicitely modelled. We have implemented these concepts in our AToM ${ }^{3}$ tool following the "model everything" philosophy. To illustrate our approach, we have modelled the TimedTraffic formalism dedicated to vehicle traffic network modelling. The syntax of TimedTraffic was meta-modelled and the operational semantics was modelled using a Graph Grammar. We also indicated how a host of formalisms and transformations can be modelled to support answering different types of questions about domain-specific models.

The main contribution of the paper is that it shows that modelling a domain-specific problem elegantly and efficiently is possible. This enables users of specific modelling formalisms to design specific applications, with relatively minimal effort, Current and future work addresses model evolution and multi-view modelling, by means of Triple Graph Grammars. We are also modelling and generating a new web-based (SVG/Ajax) user interface for $\mathrm{AToM}^{3}$ which should lower the threshold for the "model everything" philosophy.

\section{Acknowledgments}

The Natural Sciences and Engineering Research Council (NSERC) of Canada is gratefully acknowledged for partial support of this work. We acknowledge the detailed and helpful comments of the anonymous reviewers.

\section{References}

1. Harel, D., Rumpe, B.: Modeling languages: Syntax, semantics and all that stuff, part i: The basic stuff. Technical report, Jerusalem, Israel (2000)

2. Giese, H., Levendovszky, T., Vangheluwe, H.: Summary of the workshop on multi-paradigm modeling: Concepts and tools. In Kühne, T., ed.: Models in Software Engineering Workshops and Symposia at MoDELS 2006. LNCS 4364, Springer-Verlag (2006) 252 - 262

3. Costagliola, G., Lucia, A.D., Orefice, S., Polese, G.: A classification framework to support the design of visual languages. J. Vis. Lang. Comput. 13 (2002) 573-600

4. Rozenberg, G.: Handbook of Graph Grammars and Computing by Graph Transformation, Volume 1. World Scientific (1997)

5. Minas, M.: Concepts and realization of a diagram editor generator based on hypergraph transformation. Science of Computer Programming 44 (2002) 157-180

6. de Lara, J., Vangheluwe, H.: AToM ${ }^{3}$ : A tool for multi-formalism and meta-modelling. In: European Joint Conference on Theory And Practice of Software (ETAPS), Fundamental Approaches to Software Engineering (FASE). LNCS 2306, Springer (2002) 174 - 188 Grenoble, France.

7. Kelly, S., Tolvanen, J.P.: Visual domain-specific modeling: Benefits and experiences of using metacase tools. In Bezivin, J., Ernst, J., eds.: Proceedings of the International workshop on Model Engineering, ECOOP 2000. (2000) 9 pp.

8. Papacostas, C., Prevedouros, P.: Transportation Engineering and Planning. Second edn. Prentice Hall (1992)

9. Zeigler, B.P.: Theory of Modelling and Simulation. Robert E. Krieger (1984)

10. Vangheluwe, H., de Lara, J.: Domain-Specific Modelling for analysis and design of traffic networks. In Winter Simulation Conference, IEEE Computer Society Press (2004) 249 258 Washington, DC. 